\title{
A Novel Vertical Grid Transferring Technique to Prepare 3D TEM Sample from Lift-Out Sample
}

\author{
Nathan Wang, Jin Wu and Sabbas Daniel \\ Cypress Semiconductor Corporation
}

3D TEM observation denotes cross sectional viewing of an original TEM sample that provides a twodimensional image. In the semiconductor industry this method was originally applied to cases where cross sectional viewing was required but precise target location was unavailable, so the plane view TEM provides the precise location for the cross sectional one [1]. There is increasing demand now for three dimensional TEM observation because of increasing cases where feature size is smaller than the sample size and the features that have high $\mathrm{Z}$ materials mask out or obscure others [2]. 3D TEM sample preparation implies preparing a cross section from an existing TEM sample. It is always very difficult because it deals with very thin lamellas. Although it has been discussed for years [1][2][3][4][5] there is still a lack of an easy, simple and inexpensive technique. This paper introduces a novel method to prepare 3D samples from lift out (LO) samples, which does not need an Omni probe and reaches very high success rates.

A new 3D sample is a thin cross section of a previous sample so the new one is perpendicular to the original TEM grid. A very important step in preparing a 3D sample is to transfer the previous sample to a vertical grid. A TEM grid is a $3 \mathrm{~mm}$ round thin disc that fits a TEM sample holder. To transfer a micron scale sample to a grid without a micron scale tool, such as an Omni probe, is very difficult. However, it can be done if the previous sample is a LO sample. Since a LO sample sits on a sticky carbon film, the sticky carbon can land on a clean, flat surface. Such a method has been applied to transfer a LO sample to a polished sample for further ion milling [6]. The only issue here is - creating a large flat surface that is perpendicular to a TEM grid for the LO sample to land on.

A cross section of a single disc has a narrow, long area that is difficult for a LO sample to land on. A cross section of multiple discs would provide a wider area. Based on this ideal, a group of 50micron thick grids are gathered and polished to form a large flat surface (Figures 1-3). Then the LO sample is placed on the polished surface (Figure 4). The sample position and orientation are adjusted in order to position the sample right on center of one disc surface and the future sample parallel to the grid disc. The next step is to transfer the original sample to the new vertical grid by pushing the carbon film down using a probe needle of a manipulator (Figure 5). As long as the carbon film touches the solid surface it spreads over on the surface and stays with it. After disconnecting the film that the sample sits on from the mesh grid, the mesh grid can be removed. Now the sample is on a new vertical grid.

Then the stub that the sample sits on is put into a FIB chamber. Three steps in FIB are performed: First, the target location (Figure 6) is marked to indicate the precise cross section position; then FIB depositing is performed to lay a protective layer on the target location to protect the sample from ion beam damage (Figure 7); third, FIB milling is performed to prepare a electron beam transparency thin cross section for TEM observation (Figure 8).

After the sample is taken out of the FIB, the new grid that the sample sits on is then separated from the others (Figure 10). The sample is now ready for TEM observation.

This procedure is simple, easy, and inexpensive and has a very high success rate. The whole procedure normally takes less than one hour. 
An example result of applying this technique is shown in Figures 11 and 12. The TEM images revealed dislocations in silicon substrate.

\section{References}

[1] N. Wang and Sabbas Daniel, Microelectronic Failure Analysis Desk Reference 2002 suppl., ASM international, Materials Park, OH, (2002), pp.21-28

[2] K. Mcllwrath and N. Wang, ISTFA 2004, (2004), 320

[3] C.K. Liu et al, ISTFA 2002, (2002), 313

[4] T. Yaguchi, et al, Microscopy Today, 12 (6), (2004), 26

[5] J. C. Lee, et al, Microelectronics Reliability 41, (2001), 1551

[6] N. Wang, et al, Proceedings of Microscopy \& Microanalysis 2004, 1168CD

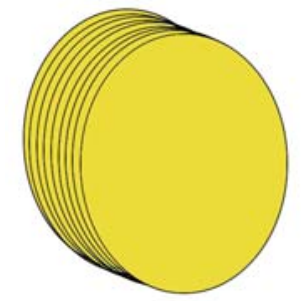

Figure 1: Gather a group of discs for polishing.

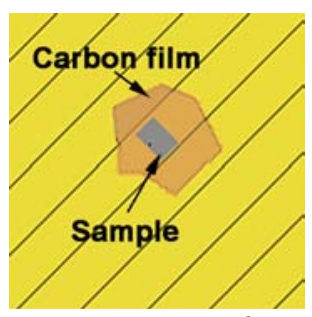

Figure 5: Use of probe needle to push the carbon film down. The sample will go with the carbon film.

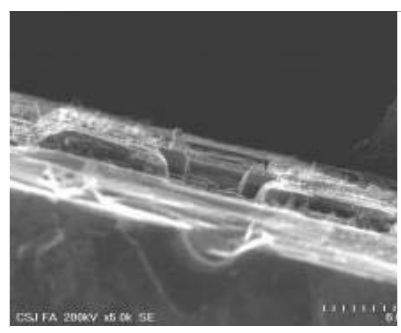

Figure 9: A SEM image showing a final sample.

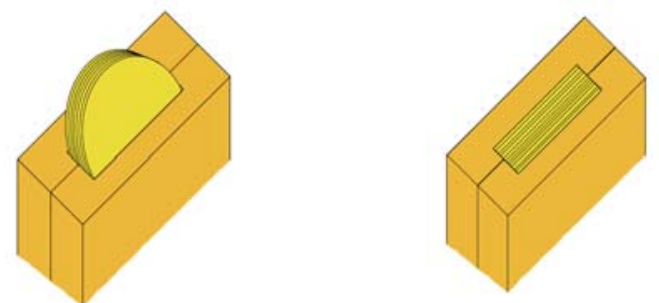

Figure 2: Discs are Figure 3: The discs are mounted on a polishing polished into half circle holder shape.

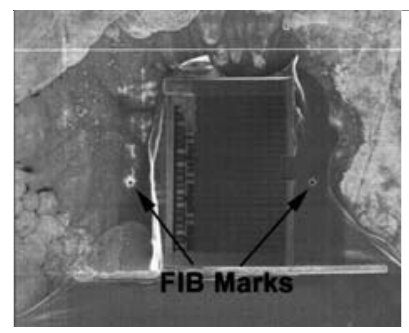

Figure 6: Use of FIB to mark the target location

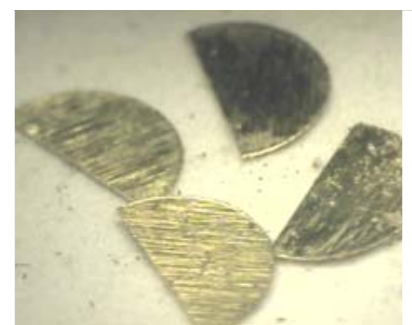

Figure 10: Separate the grids that have the $3 \mathrm{D}$ sample from others

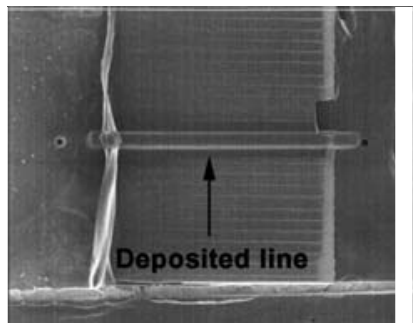

Figure 7: Use of FIB to deposite protective layer on the target location

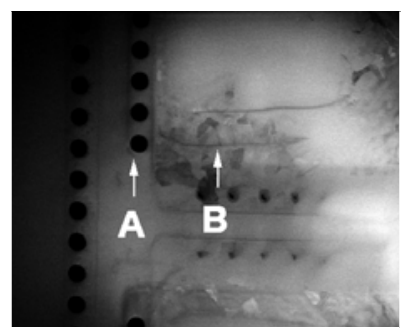

Figure 11: A plane view STEM image showing dislocations in silicon

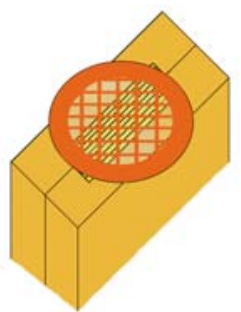

Figure 4: Lay the LO sample on the polished surface and adjust its position and orientation.

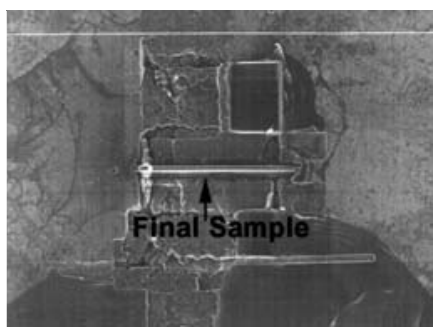

Figure 8: Use of FIB to cut the sample to final thickness

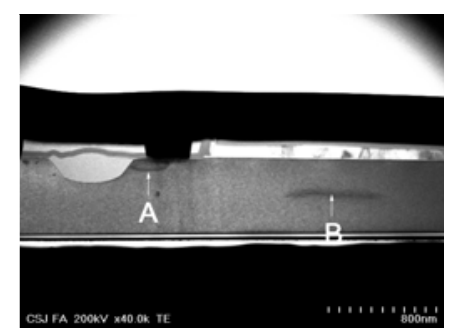

Figure 12: A cross sectional STEM image of the same sample. 